\title{
Closure of left-sided bronchopleural fistula by video-assisted mediastinoscopy: Is it always possible?
}

\author{
Nicolas Venissac, MD, Daniel Pop, MD, and Jerome Mouroux, MD, Nice, France
}

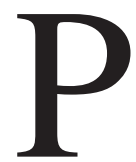

ostpneumonectomy bronchopleural fistula (BPF) is a major challenge for thoracic surgeons. Despite widespread understanding of the risk factors, the incidence varies from $0.5 \%$ to $4.5 \%$ and the mortality is still as high as $71.2 \% .^{1}$ Succesful treatment requires an individual approach in each patient. We report our experience with closure of a left-sided BPF using video-assisted mediastinoscopy (VAM), describing the technical details.

\section{Clinical Summary}

We treated 2 patients who had left pneumonectomy for lung cancer in another center. About 1 month later, the symptoms began. The chest x-ray film showed an empty pleural cavity, and a flexible fiberoptic bronchoscope identified a fistula in both cases.

The first patient started coughing up clear sputum. The physical examination and laboratory findings showed no abnormalities. The chest computed tomographic scan measured a 17.3-mm stump (Figure 1). A standard VAM technique (the equipment and instruments have been previously described ${ }^{2}$ ) was done for closure. After a short cervicotomy, the dissection began on the anterior tracheal wall: first toward the right, we liberated the right bronchus and pulmonary artery below; continuing to the left, we freed the carinal region and bronchial stump. Care was taken to avoid injuring the left pulmonary artery stump. Afterward, the left tracheal wall was dissected to liberate the tracheobronchial angle. Then, following the tracheoesophageal groove, we identified and freed the posterior aspect of the stump and carinal region. An endodissector made clear the circumference of the stump. Finally, dissection was done for a minimum 1-cm long stump. Using an endo-GIA 30 stapler (roticulator; Auto Suture Company Division, United States Surgical Corporation, Norwalk, Conn) with the knife withdrawn, we sutured the stump. A peroperative fiberoptic bronchoscopic examination showed good airtightness. The patient was discharged on the second postoperative day.

The second patient had a right pneumopathy and quickly exhibited respiratory insufficiency (Figure 2). Broad-spectrum antibiotics and a chest tube were set up. At admission, he had an arterial oxygen saturation of $92 \%$ with $15-\mathrm{L}$ oxygen flow. Blood gases were as follows: $\mathrm{PaO}_{2} 65 \mathrm{~mm} \mathrm{Hg}$ and $\mathrm{PaCO}_{2} 31 \mathrm{~mm} \mathrm{Hg}$.

From the Thoracic Surgery Department, Pasteur Hospital, Nice, France. Received for publication July 10, 2006; accepted for publication Aug 8, 2006.

Address for reprints: Nicolas Venissac, MD, Thoracic Surgery Department, Pasteur Hospital-Building H1, 30 Avenue de la Voie Romaine, 06002 Nice, France (E-mail: venissac.n@chu-nice.fr).

J Thorac Cardiovasc Surg 2006;132:1490-1

$0022-5223 / \$ 32.00$

Copyright (C) 2006 by The American Association for Thoracic Surgery doi:10.1016/j.jtcvs.2006.08.013

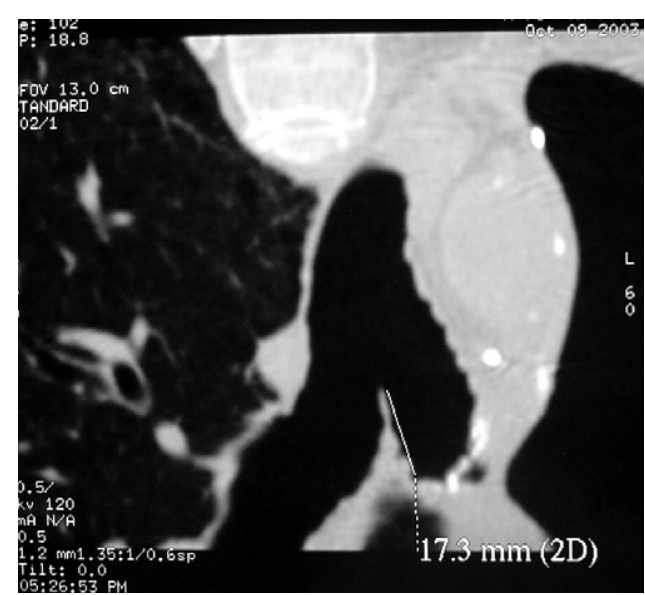

Figure 1. Chest computed tomographic scan (sagittal slice) measured the bronchial stump.

Then intubation was needed, but it was very difficult to maintain the selectivity and his condition continued to deteriorate. We did a VAM closure of the BPF and thoracoscopy to clean the pleural cavity (same operative time, patient supine). Postoperatively, his ventilatory parameters improved. Despite adapted antibiotics, the right pneumopathy did not improve, and he required increased high-pressure ventilation. Eight days later the BPF reopened, resulting in the patient's death.

\section{Discussion}

Management of BPF is difficult to assess. The best approach is to prevent it. Some basic principles to guarantee an optimal closure are as follows: insertion of sutures without tension, creation of a short bronchial stump, avoidance of excessive dissection, and gentle handling of tissues. When BPF occurs, a variety of methods have been proposed. On the right side, the bronchial stump is lacking in mediastinal coverage, which explains the difference in prevalence of right- and left-sided BPF. ${ }^{1}$ The risk is still present on the left because of the technical difficulty of creating a short bronchial stump. Suturing the BPF can be done classically by transthoracic or transpericardial sternotomy.

Azorin and associates ${ }^{3}$ were the first to report the successful closure of a left-sided 2-cm long bronchial stump using VAM. Since then, no other experience has been published elsewhere. Spaggiari ${ }^{4}$ reported a video-assisted Abruzzini technique. The right anterior parasternal mediastinotomy is assisted by left parasternal thoracoscopic access and VAM. Like other anterior approaches, this technique allows good vascular control but less exposure for the bronchial tree, especially on the left side. Second, this technique was done only in a cadaver model. The lack of bleeding, the 


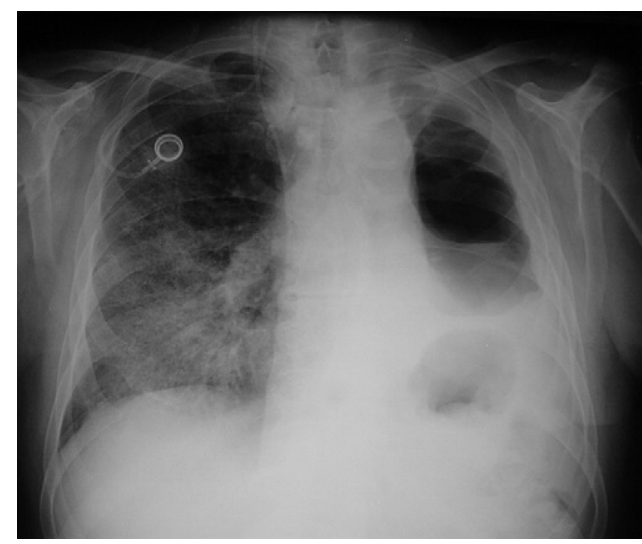

Figure 2. Chest x-ray film of patient 1 showing a left pleural cavity and right pneumopathy.

heart contractions or aortic pulsations, and the risk of opening the contralateral pleura make necessary a clinical study.

We showed the feasibiliy of VAM dissection in resecting the paratracheal mesothelial cysts. ${ }^{2}$ Our cases showed the interest of using VAM for left-sided BPF. The dissection of the trachea through its natural route enables tracheal mobilization. The mediastinal shift is not a contraindication for VAM but represents a risk for contralateral pleural opening during transpericardial sternotomy or the modified Abruzzini technique. ${ }^{4}$ Previous mediastinoscopy is not a contraindication inasmuch as the morbidity is not increased ${ }^{5}$ and there is a low risk of contamination. Our 2 cases showed good technical results. The first patient is still alive 2 years after the procedure. Unfortunately, the second patient died of severe sepsis. Perhaps all types of surgery in the presence of severe sepsis are risky.

In conclusion, each patient must be treated individually. The best method of closure must be based on the unique set of circumstances. Direct surgical repair can be achieved in most patients. The VAM technique is our choice for a long (at least $10 \mathrm{~mm}$ ) bronchial stump on the left side because its specific morbidity is minimal compared with transpericardial sternotomy or a transthoracic approach.

\section{References}

1. Asamura H, Naruke T, Tsuchiya R, Goya T, Kondo H, Suemasu K Bronchopleural fistulas associated with lung cancer operations: univariate and multivariate analysis of risk factors, management, and outcome. J Thorac Cardiovasc Surg. 1992;104:1456-64.

2. Pop D, Venissac N, Moroux J. Video-assisted mediastinoscopy: a useful technique for paratracheal mesothelial cysts. J Thorac Cardiovasc Surg. 2005;129:690-1.

3. Azorin JF, Francisci MP, Tremblay B, Larmignat P, Carvaillo D Closure of a postpneumonectomy main bronchus fistula using videoassisted mediastinal surgery. Chest. 1996;109:1097-8.

4. Spaggiari L. Video-assisted Abruzzini technique for bronchopleura fistula repair. A pathology study. J Cardiovasc Surg (Torino). 2000;41: 957-9.

5. Olsen PS, Stentoft P, Ellefsen B, Pettersson G. Re-mediastinoscopy in the assessment of resectability of lung cancer. Eur J Cardiothorac Surg. 1997;11:661-3.

\section{Gastric conduit staple line after esophagectomy: To oversew or not?}

Judith Boone, MSc, Inne H. M. Borel Rinkes, MD, PhD, and Richard van Hillegersberg, MD, PhD, Utrecht, The Netherlands

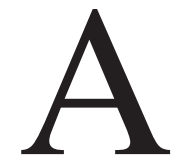

fter esophagectomy, continuity is frequently restored by means of gastric replacement. The staple line of this gastric conduit is generally oversewn to prevent leakage and erosion of adjacent tissue. This last step is often omitted during minimally invasive esophagectomy (MIE)

\footnotetext{
From the Department of Surgery, University Medical Center Utrecht, Utrecht, The Netherlands.

Received for publication July 5, 2006; accepted for publication Aug 8, 2006.

Address for reprints: Richard van Hillegersberg, $\mathrm{MD}, \mathrm{PhD}$, University Medical Center Utrecht, Department of Surgery, G04.228, Heidelberglaan 100, 3584 CX Utrecht, The Netherlands (E-mail: r.vanhillegersberg@ umcutrecht.nl).

J Thorac Cardiovasc Surg 2006;132:1491-2

$0022-5223 / \$ 32.00$

Copyright $\odot 2006$ by The American Association for Thoracic Surgery doi:10.1016/j.jtcvs.2006.08.017
}

because technical difficulties make it time consuming. ${ }^{1}$ Moreover, there is little evidence that supports the need for oversewing staple lines in gastrointestinal surgery. We describe two major complications that occurred after abandoning oversewing the staple line of the gastric conduit after esophagectomy.

\section{Clinical Summary}

PATIENT 1. A 66-year-old woman with a squamous cell carcinoma of the midesophagus underwent robot-assisted thoracoscopic esophagectomy. Through a laparotomy, the resected specimen and abdominal lymph nodes were removed. By using the GIA-stapler (GIA 80, 3.8 mm; Tyco Healthcare, Norwalk, Conn), a 3-cm-wide gastric conduit was created. ${ }^{2}$ The staple line was not oversewn.

On the first postoperative day, the left thoracic drain produced bile. Methylene blue was injected into the nasogastric tube, which was detected in the thoracic drain soon afterward. A contrastenhanced radiographic examination of the gastric conduit showed 